\title{
Radio frequency controlled microvalve for biomedical applications
}

\author{
Don W. Dissanayake, Ajay C. Tikka, Said F. Al-Sarawi and Derek Abbott \\ The Centre for High Performance Integrated Technologies and Systems (CHiPTec), School of \\ Electrical and Electronic Engineering, University of Adelaide, SA 5005
}

\begin{abstract}
In this paper we propose the use of a RF controlled microvalve for implementation on a PZT substrate for biomedical applications. Such device has a huge range of applications such as parallel mixing of photo-lithographically defined nanolitre volumes, flow control in pneumatically driven microfluidic systems and lab-on-chip applications. The microvalve makes use of direct actuation mechanisms at the microscale level to allow its use in vivo applications. A number of acoustic propagation modes are investigated and their suitability for biomedical applications, in terms of the required displacement, device size and operation frequency. A theoretical model of the Surface Acoustic Wave (SAW) device is presented and its use in micro-valve application was evaluated using ANSYS tools. Furthermore, the wireless aspect of the device is considered through combining the RF antenna with the microvalve simulation by assuming a high carrier frequency with a small peak-to-peak signal. A new microvalve structure which uses a parallel type piezoelectric bimorph actuator was designed and simulated using ANSYS tools. Then, further optimization of the device was carried out to achieve a better coupling between electrical signal and mechanical actuation within the SAW device.
\end{abstract}

Keywords: microvalve, micropump, SAW, biomedical, ANSYS, Implantable devices, actuators

\section{INTRODUCTION}

Microvalves and micropumps are of greater interest when it comes to nano-litre drug delivery in biomedical applications. The manipulation of the fluid flow at nano-litre scale is a fundamental function that has a wide range of applications such as parallel mixing of photo-lithographically defined nanolitre volumes, flow control in pneumatically driven microfluidic systems, lab-on-chip applications, precision manufacturing, drug delivery and miniaturisation of chemical and bioanalysis systems. It is proven that microvalves are essential components of these miniaturised fluidic systems [1].

The importance of a wirelessly controlled implantable microvalve for biological applications is increasing gradually. The need of a passive microvalve which can be used efficiently and effectively for biomedical applications is highly regarded. The common requirements by these applications are small in size, a minimal amount of power to operate and biocompatible with human body. The important aspects of these devices are discussed in the flowing sections for a microvalve which needs to be bio-friendly, remotely sensed and actuated, battery-less, security coded and possible to fit in small body cavities.

In section 2, a review of device operation is provided in addition to power transfer from an interrogating RF signal to the microvalve. Section 3, provides a review of different micropump structures and an analysis of different actuation schemes. Device modeling of SAW device modes are discussed in section 4. Based on the developed model, section 5 provides device simulation using ANSYS tools. As the input power directly affect the device actuation, section 6 discusses the tradeoffs between operating frequency and RF signal penetration in the human skin. Section 7 presents a designed microvalve structure with a comparison of the performance of the microvalve.

\section{DEVICE OPERATION}

In order to supply the required power to the microvalve to cause an actuation, a power source is needed. Some implanted devices are powered, for some short term applications, using a battery or some other devices that utilise inductive coupling to charge a capacitor for a burst operation [2]. There are obvious disadvantages with implanting an energy source with an implantable biomedical device. These include the need for source replacement, extra mass and size of the microvalve. It is apparent that the number of useful biomedical applications would greatly increase if the energy source was not integrated with the microvalve.

Smart Materials IV, edited by Nicolas H. Voelcker, Proc. of SPIE Vol. 6413, 64130D, (2006)

0277-786X/06/\$15 - doi: 10.1117/12.695743

Proc. of SPIE Vol. 6413 64130D-1 
Successful realization of a SAW-Interdigital Transducer (IDT) based passive (battery less) wireless sensor is found in the literature [3]. The developed sensor can be used in implants such as in the neonatal intensive care units to continuously monitor the neonatal newborns, causing minimal discomfort for the newborns. This particular sensor relies on modulating the load impedance. However such an approach is not suitable for the proposed microvalve design because it is expected to use the output from the SAW device to power the actuator. Considering a generalized application domain, researchers have addressed the possibility of using biomedical implants without batteries for biotelemetry applications and suggest a different principle using the converse piezoelectric effect, to control the microvalve without batteries [4]. The proposed device structure is based on this converse piezoelectric effect combined with a SAW device.

\subsection{Wireless Operation}

In implantable applications, it is important that the device not to be triggered by spurious RF signals. One possible way to overcome this issue is to design the system to operate in the near field, to operate only when the external RF source is in near proximity. The strength of the electromagnetic coupling between the antennas affects the telemetry distance between the sensor antenna and the control unit antenna [3]. However the electromagnetic coupling greatly depends on the antenna geometry, size and directivity.

For a microvalve consisting of a SAW device, the required mechanical actuation forces can be typically of the order of $100 \mu \mathrm{N}$ [5] and a $10 \mu \mathrm{m}$ displacement corresponds to nano-Jules of energy. This suggests that the power requirement for the external RF source at typical frequencies is extremely small. Moreover the system can be designed to work when the $\mathrm{RF}$ source is closer to the user or even in contact with the skin. In some applications there is a need to have more power delivered to the antenna hence an efficient antenna would be needed. However if the distance between the interrogator antenna and the sensor antenna is short, a slightly inefficient antennas can be used without much loss in performance. Consequently less effort and cost is required for the design and fabrication of the antenna [5].

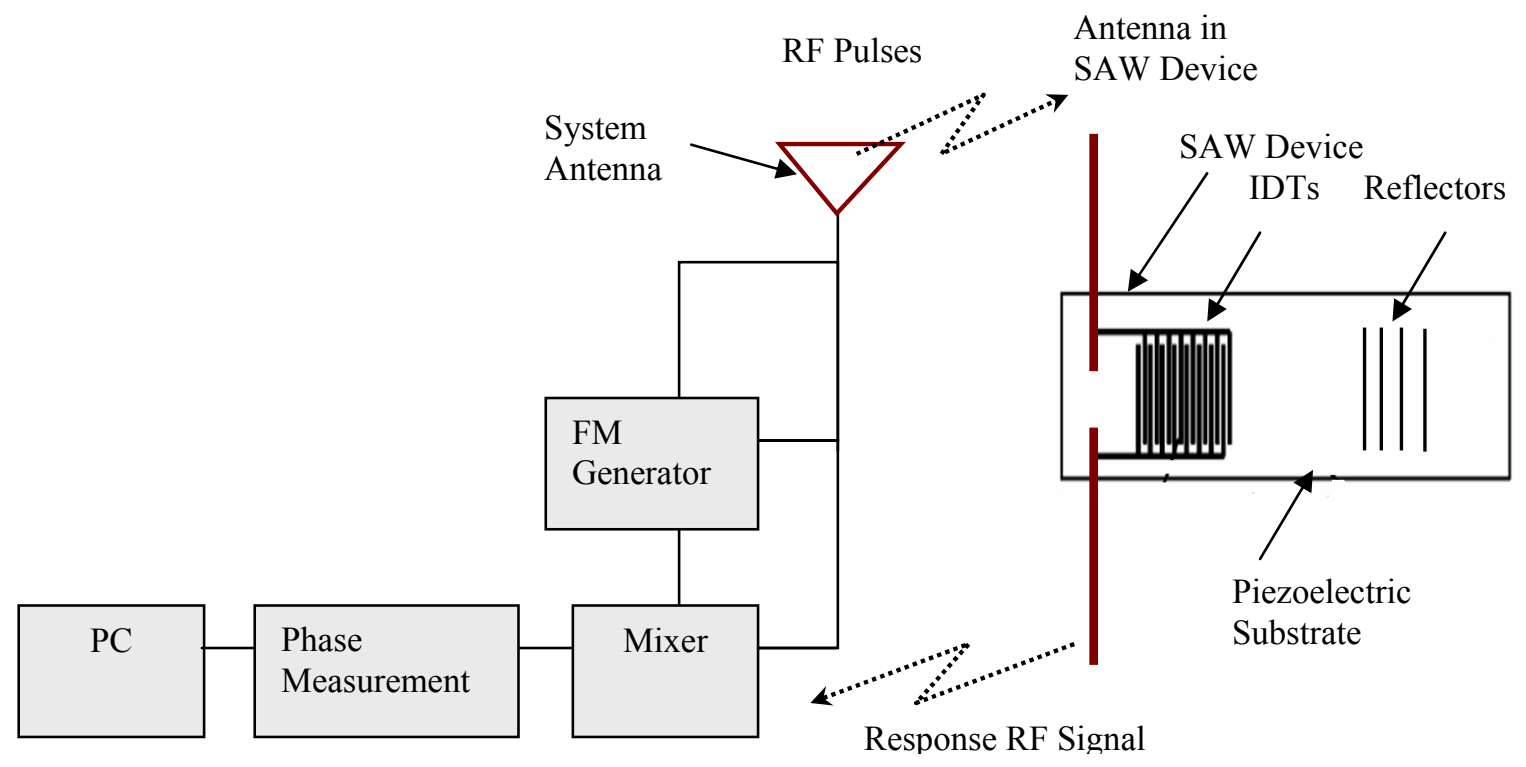

Figure 1. Direct actuation method implementation: a SAW device directly connected to a micro-antenna (through IDTs) is used to establish the wireless connection for the microvalve.

Figure 1 shows a setup for interrogating a SAW device with an integrated antenna. The received RF signal power by the device is directly used to generate a SAW in the device which is then used to actuate a piezoelectric actuator in a latter stage. In order to interrogate the microvalve, it is important to design a near proximity operation of a wireless system. Passive and wireless operations are desirable in applications where an unrestricted operation is more critical than the long distance communication [3]. The ability to store energy in SAW and the possibility of handling high frequencies enables the construction of passive sensors with the capability to be interrogated by RF signals [6]. 


\section{A REVIEW OF MICROVALVES/MICROPUMPS AND ACTUATION METHODS}

Analysis of currently available Micro Electro Mechanical Systems (MEMS) structures for microvalves and micropumps is significant in designing novel microvalve structures. It is beneficial to incorporate the pumping effect in to the microvalve design as it reduces the dependency of the operation of the device on the surrounding fluid flow. It is being argued that the most promising micro-fluidic products are devices for DNA, protein analysis and drug delivery [7-10], which implies that the development of a biocompatible microvalve with advanced functionality, will be very much useful for a host of applications as mentioned previously.

\subsection{Different Microvalve / Micropump Analysis}

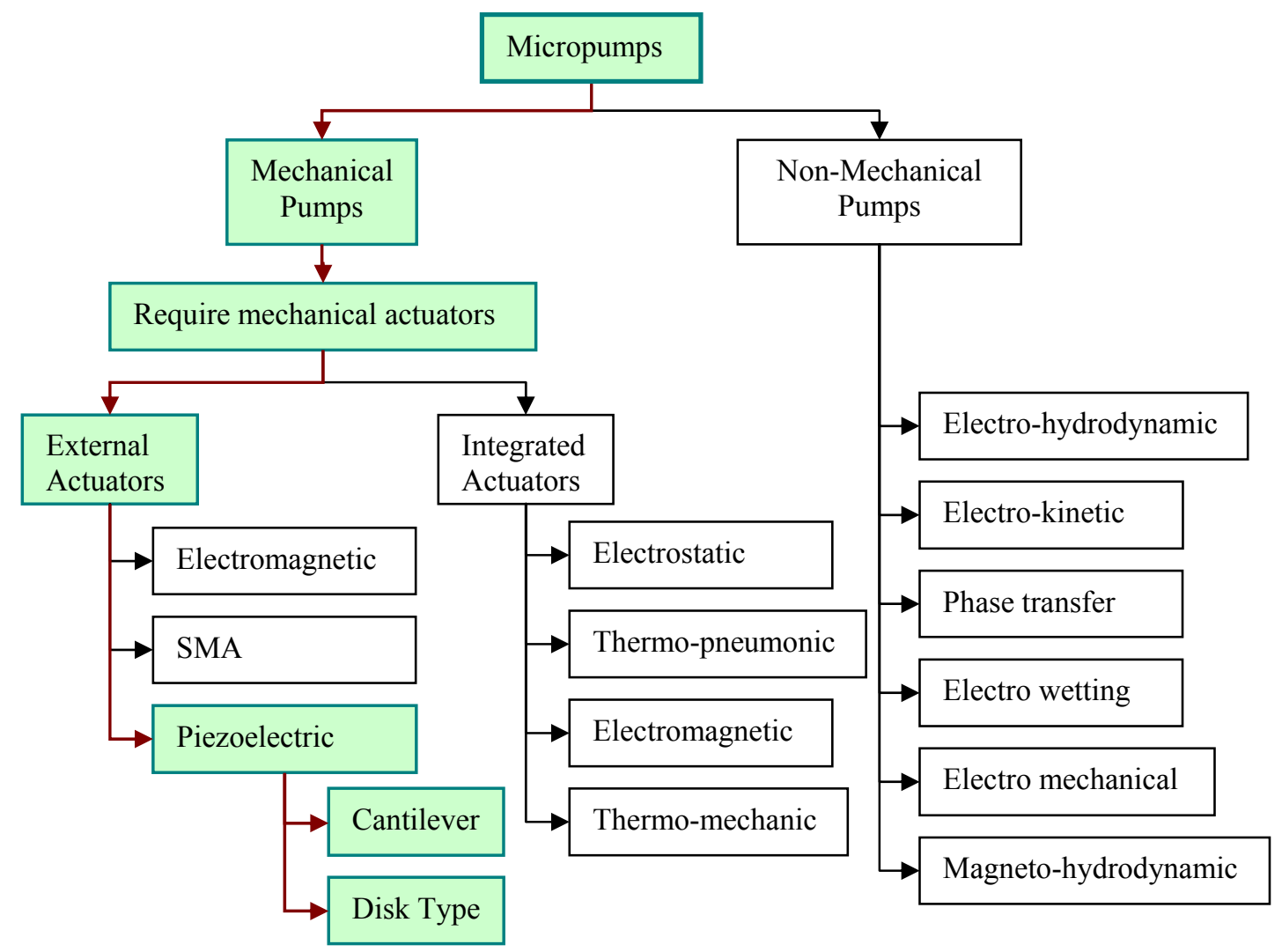

Figure 2. Micropump analysis based on their operation and actuation method. Only the major actuation mechanisms are considered.

Different types of micropumps are analyzed based on their actuation method and basic operation and a classification is presented in Figure 2. Micropumps can be mainly classified as mechanical and non-mechanical micropumps. Mechanical pumps usually utilize moving parts such as check valves, oscillating membranes, or turbines for delivering constant fluid volume in each pump cycle. Most mechanical pumps require a mechanical actuator which generally converts an electrical energy into a mechanical work. Mechanical actuators can be either external or integrated actuators. External actuators consist of an actuator separate to the micropump body where as integrated actuators are micro-machined to the micropump.

Different characteristics such as flow rates versus pump size, energy required for a single stroke versus pump size, Reynolds number for different pump structures and back pressure versus flow rate for different micropumps is presented in the literature [7]. It is evident from these analyses that in general mechanical pumps are larger in size than nonmechanical pumps. Moreover, it shows that most mechanical micropumps have achieved flow rated between $10 \mu \mathrm{L} / \mathrm{min}$ and $1000 \mu \mathrm{L} / \mathrm{min}$ and no mechanical micropumps have been able to generate a flow rate less than $1 \mu \mathrm{L} / \mathrm{min}$ accurately. This occurs due to the large viscus force and their relatively large size. However some non-mechanical pumps have 
achieved the flow rate limit of $1 \mu \mathrm{L} / \mathrm{min}$ accurately. These results highlights the issue of mechanical scaling law has to be followed carefully when designing micropumps. Fluid flow rate, required pump energy or the mechanical work and the pump size are the main factors to be considered under mechanical scaling law.

\subsection{SAW and Piezoelectric Actuation}

Various different actuation schemes are widely used for microvalves and micropumps [1,3,7,8-10]. Different actuation schemes are discussed to identify which actuation mechanism is best suited for the application domain in concern. Analysis of different actuation mechanisms for microvalve designs can be found in the literature $[1,7,10]$. Namely they are piezoelectric, electrostatic, magnetic, pneumatic and thermal actuators. Magnetic actuators can be further classified in to electromagnetic and solenoid-plunger while thermal actuators can be either thermo-pneumatic, bimetallic or shape memory alloy (SMA). Much more comparison is discussed for different actuation mechanisms in [9] with relevant to microvalve design for fuel cell applications. Table 1 below shows a comparison of force, displacement, response time and reliability for different actuation mechanisms.

Table 1. Comparison of different actuation mechanisms considering Force, Displacement, Response time and Reliability [9].

\begin{tabular}{|l|l|l|l|l|}
\hline Actuators & Force & Displacement & Response Time & Reliability \\
\hline Solenoid-plunger & Small & Large & Medium & Good \\
\hline Piezoelectric & Very Large & Medium & Fast & Good \\
\hline Pneumatic & Large & Very Small & Slow & Good \\
\hline SMA & Large & Large & Slow & Poor \\
\hline Electrostatic & Small & Very Small & Very Fast & Very Good \\
\hline Thermo-pneumatic & Large & Medium & Medium & Good \\
\hline Electromagnetic & Small & Large & Fast & Good \\
\hline Bimetallic & Large & Small & Medium & Poor \\
\hline
\end{tabular}

For a microvalve be used in biomedical applications, the device should be highly reliable and should have a better response time to gain a precise control as much as possible. Moreover a higher force is also an advantage if the microvalve or the micropump is surrounded by a high viscus fluid, so that the actuators can work well against the pressure generated by the fluid. In an ideal case a higher displacement is highly expected.

The major drawback of external actuators is large size which restricts the overall size of the micro-pump. While the biggest advantage of them is the generation of a relatively large force and displacement [10]. Despite the fast response time and good reliability, electrostatic actuators cause small force and very small stroke [7]. However piezoelectric actuators stand out for their low power consumption and high force generation [8], despite their complex fabrication requirements and medium output displacement. Further more for pyroelectric actuation, a few degrees of temperature difference is required to gain a better micro level displacement [8] which would not be ideal for an implantable microvalve. Considering factors discussed above, the piezoelectric actuation is considered to be the best choice for the application domain in concern along side SAW devices. 


\section{MODELING BACKGROUND FOR SAW MODES}

\subsection{FEA of Acoustic Wave propagation}

The finite-element method is powerful in numerical analysis because it can be used to model arbitrary geometry and anisotropic material properties. Piezoelectric materials are anisotropic and the elastic deformation in such materials is coupled with the electric field. The constitutive tensor equations for the piezoelectric materials are:

$$
\begin{aligned}
& \mathbf{T}_{i j}=\mathbf{c}_{i j k l}^{E} \mathbf{S}_{k l}-\mathbf{e}_{k i j} \mathbf{E}_{k}, \\
& \mathbf{D}_{i}=\mathbf{e}_{i k l} \mathbf{S}_{k l}-\varepsilon_{i k}^{S} \mathbf{E}_{k},
\end{aligned}
$$

Where $\mathbf{T}_{i j}$ is the mechanical stress tensor, $\mathbf{S}_{k l}$ is the mechanical strain, $\mathbf{E}_{k}$ is the electric field vector, $\mathbf{D}_{i}$ is the dielectric displacement, $\mathbf{c}_{i j k l}^{E}$ is the stiffness tensor for constant electric field, $\mathbf{e}_{k i j}$ is the piezoelectric coupling tensor and $\varepsilon_{i k}^{S}$ is the permittivity tensor for constant strain. The relationship between the electric field $\mathbf{E}$ and the electric potential $\phi$ is:

$$
\mathbf{E}_{i}=-\frac{\partial \phi}{\partial x_{i}}
$$

Mechanical strain $\mathbf{S}$ and mechanical displacement $\boldsymbol{u}$ are related by:

$$
\mathbf{S}_{i j}=\frac{1}{2}\left[\frac{\partial \boldsymbol{u}_{i}}{\partial x_{j}}+\frac{\partial \boldsymbol{u}_{j}}{\partial x_{i}}+\left(\frac{\partial \boldsymbol{u}_{k}}{\partial x_{i}} \cdot \frac{\partial \boldsymbol{u}_{k}}{\partial x_{j}}\right)\right] \text { And }\left(\frac{\partial \boldsymbol{u}_{k}}{\partial x_{i}} \cdot \frac{\partial \boldsymbol{u}_{k}}{\partial x_{j}}\right) \rightarrow 0 \quad \therefore \mathbf{S}_{i j}=\frac{1}{2}\left[\frac{\partial \boldsymbol{u}_{i}}{\partial x_{j}}+\frac{\partial \boldsymbol{u}_{j}}{\partial x_{i}}\right] \text {, }
$$

Newton and Maxwell's laws define the elastic and electrical behavior of piezoelectric materials respectively, where $\rho$ is the mass density of the material.

$$
\begin{gathered}
\frac{\partial \mathbf{T}_{i j}}{\partial x_{j}}=\rho\left(\frac{\partial^{2} \boldsymbol{u}_{i}}{\partial t^{2}}-\mathbf{F}_{i}\right) \mathbf{F}_{i}=0 \text { (Forces acting on the body per unit mass) } \therefore \frac{\partial \mathbf{T}_{i j}}{\partial x_{j}}=\rho \frac{\partial^{2} \boldsymbol{u}_{i}}{\partial t^{2}} \\
\nabla . \mathbf{D}=0 \text { (With the absence of intrinsic charge) }
\end{gathered}
$$

Eqns (1) to (6) can be reduced to the following relationships:

$$
\begin{gathered}
\mathbf{c}_{i j k l}^{E} \frac{\partial^{2} \boldsymbol{u}_{l}}{\partial x_{j} \partial x_{k}}+\mathbf{e}_{k i j} \frac{\partial^{2} \phi}{\partial x_{j} \partial x_{k}}=\rho \frac{\partial^{2} \boldsymbol{u}_{i}}{\partial t^{2}} \\
\mathbf{e}_{j k l} \frac{\partial^{2} \boldsymbol{u}_{l}}{\partial x_{j} \partial x_{k}}-\varepsilon_{i k}^{S} \frac{\partial^{2} \phi}{\partial x_{j} \partial x_{k}}=0
\end{gathered}
$$

Eqns (7) and (8) can be further reduces to a system of four coupled wave equation for the electric potential and the three components of the displacement in the piezoelectric substrate. The values of these electrical and mechanical quantities at arbitrary positions on the piezoelectric element are given by a linear combination of polynomial interpolation function as:

$$
\begin{gathered}
\boldsymbol{u}_{i}(x)=N_{a}(x) \boldsymbol{u}_{i a}, \\
\phi(x)=N_{a}(x) \phi_{a},
\end{gathered}
$$


Where $\boldsymbol{u}_{i a}$ is the component of the displacement and $\phi_{a}$ is the electrical potential at the nodes of an element and $N_{a}(x)$ is the shape functions of the elements. The index $a$ is the node number in an element.

\subsection{SAW Propagation Modes}

A SAW is a sound wave that propagates along the surface of a solid and is contained within the solid. There are a number of propagation modes in a SAW device. Some of these modes are Rayleigh waves, Shear Horizontal (SH) waves, Lamb waves and Love waves. The selection of a propagation mode depends on the type of material and the intended application. Different crystal cuts can be used to generate a specific surface acoustic mode [11-16].

Rayleigh mode is the typical wave propagation mode in a SAW device. However, if a SAW sensor or actuator is in a fluid environment, it is well known that the Rayleigh waves are quickly damped- therefore would not be effective. This is due to the fact that the particles have a surface normal displacement component as well as a surface parallel component. The use of horizontal wave modes is identified as the solution to avoid damping [13]. Shear Horizontal (SH) wave modes are commonly used in fluidic environments, since SH waves subject to lesser damping in this sort of applications. The appropriate horizontal modes are ensured by the appropriate material properties and the correct manufacture of the multifunction polymer [13]. Based on variety of SAW devices that have been designed and fabricated Lamb waves have been used for sensing impact damage, cracks, delamination and corrosion. Where as Love waves have been found to be ideal for detection of ice formation, monitor the onset of crack formation and crack propagation. Moreover Rayleigh waves have been used for sensing deflection, strain, temperature humidity, pressure and acceleration $[11,12]$.

\section{SAW DEVICE SIMULATION}

In order to get an understanding of how a SAW device behave when it is operating as a sensor and/or actuator, SAW devices with different material properties [17-19] and different crystal cuts [14-16] are designed and animated in ANSYS. Figure 3(a) shows the geometry of a simple SAW device which consists of a piezoelectric substrate and a set of IDTs. Two output electrodes are used to observe the output voltage for a high frequency sinusoidal input voltage. Figure 3(b) shows the meshed SAW device in ANSYS and this is the stage before the solution phase in ANSYS. In order to carry out the FEA in ANSYS, it is required to mesh the developed geometry and allocate necessary boundary conditions and other actuation forces such as input voltage [19]. These sorts of simulations are beneficial in finding out the best suited materials for a specific application and provide an estimate of power requirements and insertion loss associated with SAW devices.

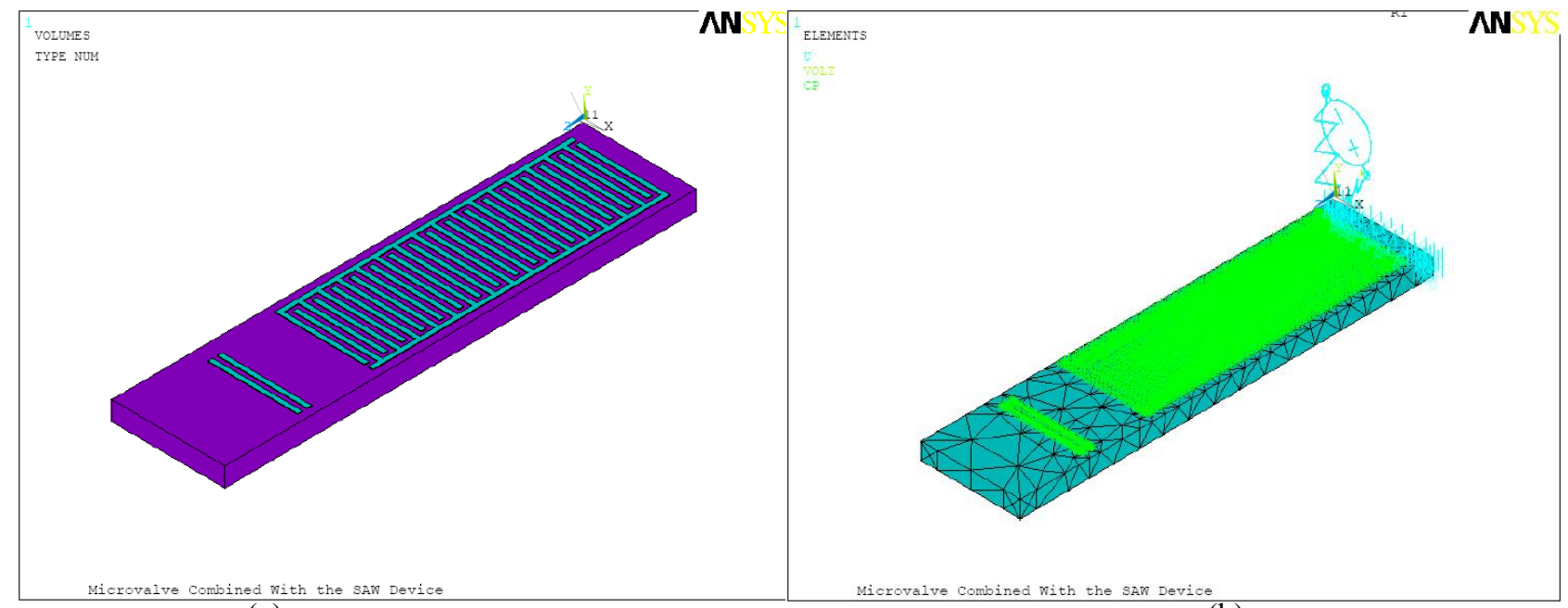

(a)

(b)

Figure 3. (a) SAW Geometry consists of a piezoelectric substrate and a set of IDTs with 16 fingers for each IDT, Meshed and boundary condition allocated SAW device-ready for analysis. The RF signal is emulated by a high frequency sinusoidal input voltage signal. Dimensions of the device are $2.5 \mathrm{~mm}(\mathrm{~L}) \times 600 \mu \mathrm{m}(\mathrm{W}) \times 100 \mu \mathrm{m}(\mathrm{H})$. 
Power tolerance of SAW devices is also an important aspect to consider, in improving the performance of the battery less device. A few different approaches can be identified regarding power tolerance in SAW devices. One such approach is to use multilayer structures or epitaxial growth to improve the metal deposition. Epitaxial growth is the growth of one layer of crystals on another such that they have the same structure [21]. Another approach is to reduce the temperature in order to minimize the stress induced material transport (acoustomigration) in the SAW device. Yet another approach is to optimize the acoustic structure in order to improve power durability by reducing the amount of stress in the electrodes [20]. The important outcome to consider is above approaches represent complementary tradeoffs in achieving optimal performance and the power handling requirements become more prominent with the increasing frequency.

\section{TRADEOFFS IN ANTENNA DESIGN}

Designing antennas for embedded applications is a challenge because of the factors such as reduced antenna efficiency, impact of the environment on the antenna, the need to reduce the antenna size and the very strong effect of multi-path losses. Designing implantable microstrip antennas with focus on antenna sizing, operating frequency and biocompatibility is discussed in this section. To improve the uniformity of radiation from embedded antennas, nonuniform insulation is suggested and microstrip designs are chosen because of their huge flexibility in design, conformability and shape [22].

\subsection{Effect on Antenna Size and Resonance Frequency}

The antenna sizing can be reduced for a given frequency by the addition of feed and ground points and properly locating them on the antenna. The bandwidth of the antenna is proportionate to the distance between feed and ground points [22] hence provide a chance to fine tune the antenna specifications.

Use of materials with high dielectric constants is another method to reduce the antenna size. But the consequence is they tend to have higher losses due to the surface wave and therefore poor efficiency. The skin tissue attenuates the power of $\mathrm{RF}$ signals due to the skin effect [23] and the propagation loss is proportionate to the signal frequency. As a result, it develops a lot of design issues in micro-antenna design and development. However, high dielectric constant of the human body tissue reduces the wavelength near the antenna and hence the physical size [2]. Also the superstrate material cause a reduction of the power deposited in the body near the antenna. Working along with the skin effect, one other method to reduce the size of the antenna is to place superstrate material only over the areas with high current density. However, the propagation losses in biological tissues can be countered by the gains in the efficiency of compact antennas as their electric size increases [2].

Effect of the antenna parameters on the antenna resonance frequency is another important area to dive into. Because an interrogation frequency has to be selected considering a lot of antenna parameters and other reference power levels accepted for human exposure [24]. The shape of the antenna affects the resonance frequency of an antenna and higher resonant frequency is achievable from electrically shorter antennas. As the permittivity of the substrate and superstrate materials increases, the resonance frequency decreases because of the resulting shorter wavelength. However as the thickness of the substrate increases, the effective dielectric constant also increases. As a result the antenna appears electrically longer and hence has a slightly lower resonance frequency. In contrast, for an increased superstrate thickness, the effective permittivity is reduced by insulating the antenna from higher dielectric body material and results in an increased resonance frequency [23].

\subsection{Tradeoff between Antenna Size and Frequency}

A tradeoff between the operating RF signal and the antenna size for wireless microvalves are discussed in this section. Such trade-offs determine the amount of power that can be delivered to the microvalve for actuation. Since the approach is to obtain power for the microvalve actuator directly from the interrogating RF signal, it is extremely important to analyse and determine the power levels that the microvalve antenna receives from the transmitter antenna at the control centre. The relationship between the operating radiofrequency $(f)$ and the wavelength $(\lambda)$ of the signal is:

$$
\lambda=\frac{c}{f},
$$




$$
\text { and } \quad l=\frac{\lambda}{x} \Rightarrow l=\frac{c}{x f},
$$

Where $c$ is the speed of light $\left(3 \times 10^{8} \mathrm{~m} / \mathrm{s}\right)$ and $x$ is taken to be 16 assuming the analysis is for a simple dipole antenna. Based on Eqn (12) the inverse relationship between the antenna size and the interrogating signal frequency is seen from Figure 4. More importantly this analysis shows the significance of choosing an operating frequency for the wireless communication, while keeping the size of the antenna within the specifications. But there are other major factors which have to be considered in arriving at an appropriate RF signal for the antenna.

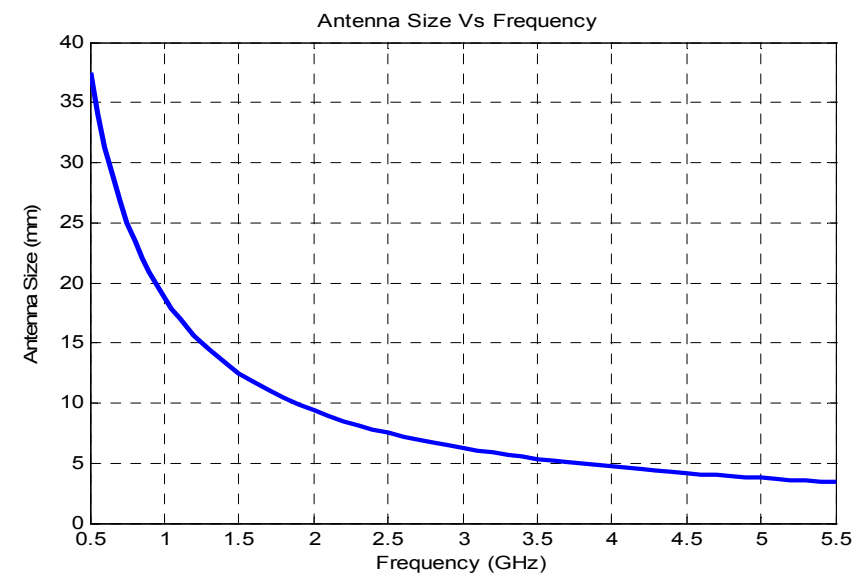

Figure 4. Antenna Size Vs Frequency Analysis for a simple dipole antenna, $x$ is taken to be 16 for this analysis

One of them is the effect on the signal strength due to the skin effect cause by the body tissue. When an electromagnetic signal propagates through human tissue, the power density associated with the signal attenuates exponentially due to the skin effect. The skin effect is defined as the tendency of a high frequency alternating current to distribute near the surface of the conductor. As a result the current density near the surface of the conductor is higher than that at the middle of the conductor. Consequently the term penetration depth for a material can be defined as the point where the signal amplitude reduces by $37 \%(1 / \mathrm{e})$ or the signal power density reduces by $13 \%\left(1 / \mathrm{e}^{2}\right)$ from the incident signal power at the surface of the skin [23]. An analysis is shown below considering the effect of attenuation of the signal due to the skin effect.

For preliminary calculations, the length of the antenna is assumed to be less than $10 \mathrm{~cm}$. The required maximum energy for the actuator is taken to be $100 \mathrm{~nJ}$ [4]. The expected operating frequency of the actuator is taken to be less than $50 \mathrm{~Hz}$ because a lower operating frequency provides a better modulation of the fluid flow and results in achieving better fluid flow rates. The effective area of the antenna is chosen to be less than $1 \mathrm{~cm}^{2}$ to be compatible with biomedical implants. Based on the above parameters the following calculation is carried out.

Required power for the actuator

$$
\begin{array}{ll}
\text { Required power for the actuator } & =\text { Energy per cycle*number of cycles* safety margin } \\
& =100(\mathrm{~nJ}) * 50(\mathrm{~Hz}) * 4 \text { (where } 4 \text { is the safety margin) } \\
& =20 \mu \mathrm{W} \\
\text { The power density }(S) \text { at the antenna } & =\text { required power/ effective area of the antenna } \\
& =20(\mu \mathrm{W}) / 1\left(\mathrm{~cm}^{2}\right) \\
& =0.2 \mathrm{~W} / \mathrm{m}^{2}
\end{array}
$$

Relationship between the power density at the implanted antenna $(S)$ and the penetration depth $(\delta)$ is given by

$$
S o=S * e^{(2 * d / \delta)}
$$


Where, $S o$ is the power density at the surface of the skin tissue and $d$ is the distance to the implanted antenna from the surface of the skin tissue. Based on the calculation and the Eqn (13) above and using relevant penetration depths for different frequencies as shown in Table 2, power density required at the surface of the skin $\left(S_{o}\right)$ for different penetration depths are plotted for a constant $S$ of $0.2 \mathrm{~W} / \mathrm{m}^{2}$. Graphs in Figure 5 show the behavior obtained and how rapidly power densities change for higher frequencies. Moreover international regulations on occupational exposure to time varying electromagnetic fields are followed when selecting a frequency [24].

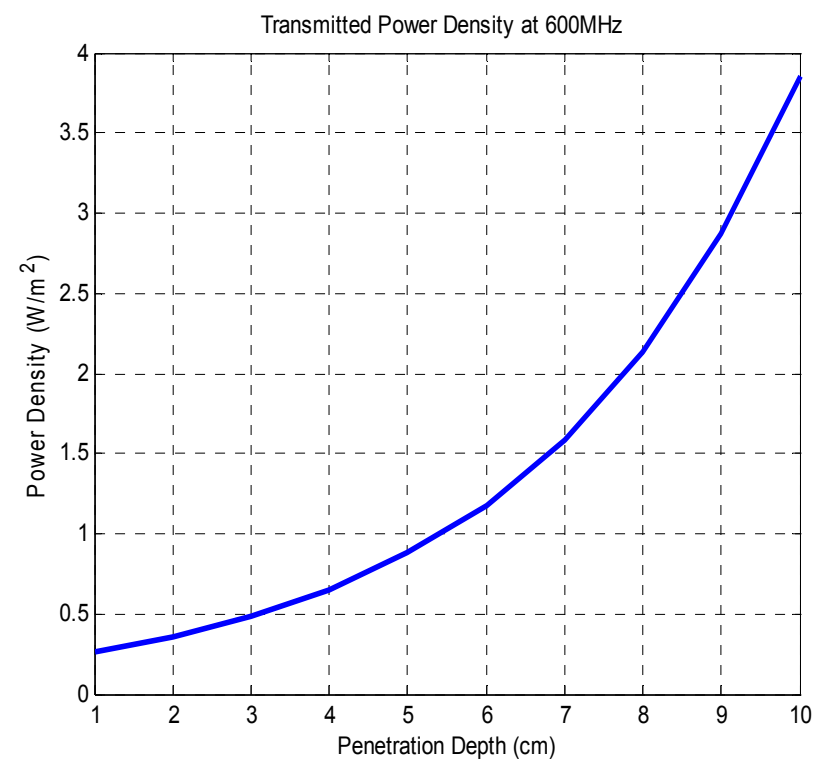

(a)

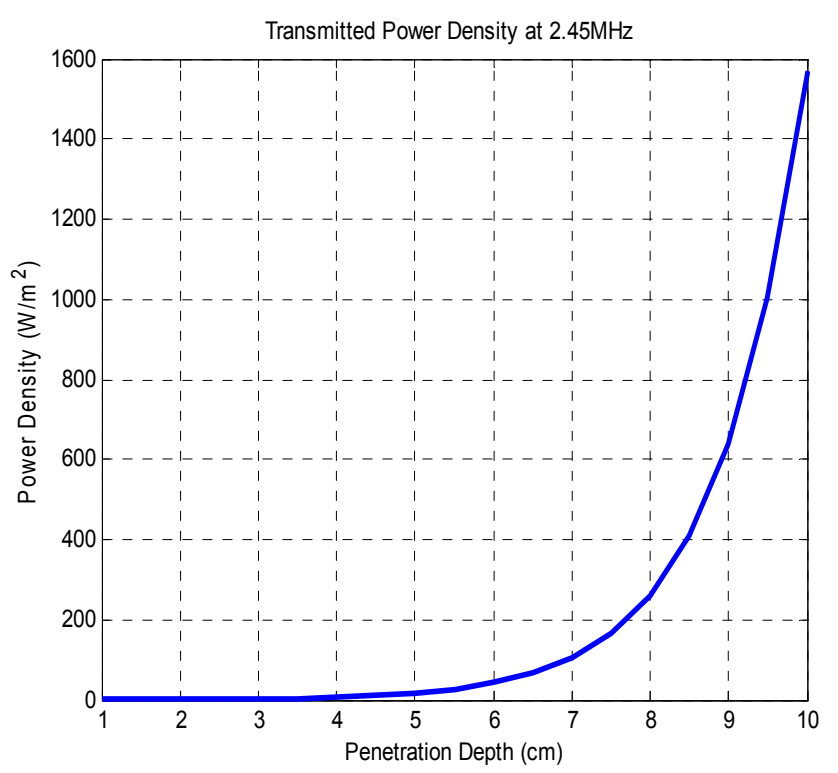

(c)

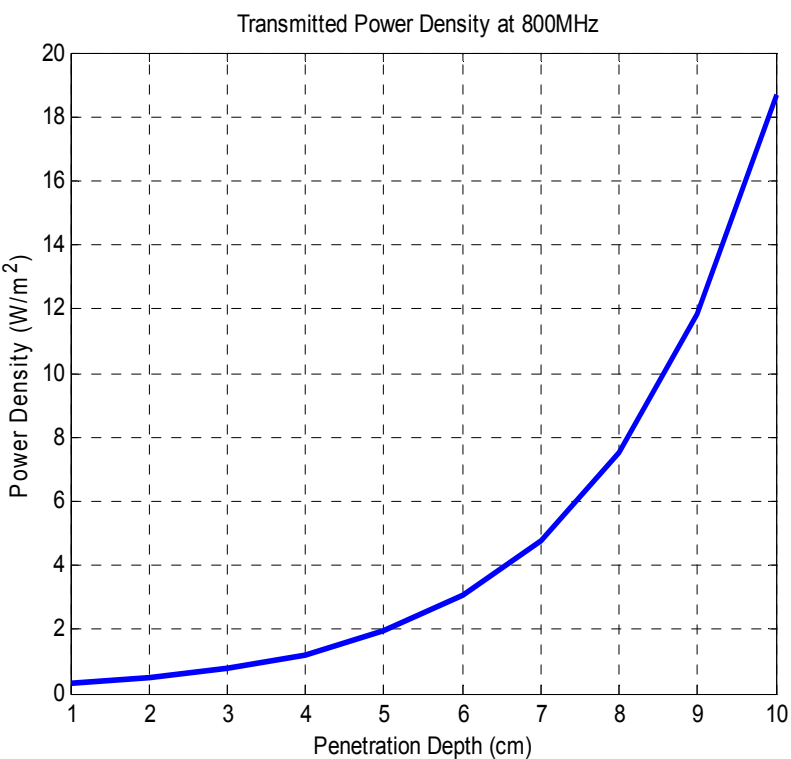

(b)

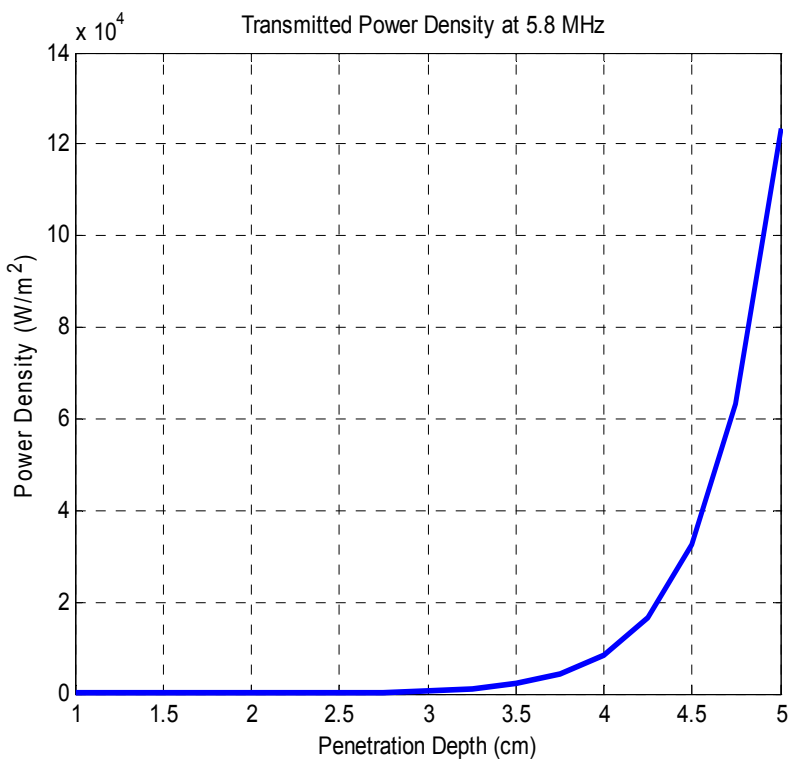

(d)

Figure 5. Different power densities which reach the surface of the skin tissue for different frequencies as the implanted antenna is placed at different distances from the skin. Transmitted power density at (a) $f=600 \mathrm{MHz}$ and $\delta=67.57 \mathrm{~mm}$ (b) $f=800 \mathrm{MHz}$ and $\delta=44.09 \mathrm{~mm}$ (c) $f=2.45 \mathrm{GHz}$ and $\delta=22.30 \mathrm{~mm}$ (d) $f=5.8 \mathrm{GHz}$ and $\delta=7.5 \mathrm{~mm}$. 
Table 2. Maximum allowed reference power densities and penetration depths for skin tissue at different ISM frequencies [24].

\begin{tabular}{|c|c|c|}
\hline Frequency $(\mathrm{GHz})$ & $\begin{array}{c}\text { Maximum allowed reference } \\
\text { Power Density }\left(\mathrm{W} / \mathrm{m}^{2}\right)\end{array}$ & Penetration Depth $(\mathrm{mm})$ \\
\hline 0.60 & 15 & 67.57 \\
\hline 0.80 & 20 & 44.09 \\
\hline 2.45 & 50 & 22.30 \\
\hline 5.80 & 50 & 7.50 \\
\hline
\end{tabular}

Considering results in Figure 5 and reference power levels shown in Table 2, frequencies $2.45 \mathrm{GHz}$ and $5.8 \mathrm{GHz}$ are eliminated from the consideration. This is because to operate the microvalve which could be implanted in a few centimeters from the skin requires power densities much higher than $50 \mathrm{~W} / \mathrm{m}^{2}$ hence it violates reference levels. Moreover according to Figure 4, lower the antenna frequency higher the antenna size is. Therefore lower frequencies are also eliminated for the consideration. Consequently this analysis shows a frequency around $1 \mathrm{GHz}$ would be ideal for the operation of the implanted microvalve.

\section{MICROVALVE STRUCTURES}

A few different microvalve structures were designed using ANSYS. A parallel type piezoelectric bimorph actuator is used to modulate the fluid flow since it generates a higher force as well as a better displacement over a series type piezoelectric bimorph actuator [26,27] and a unimorph actuator. The actuator dimensions are $4 \mathrm{~mm}(L) \times 800 \mu \mathrm{m}(W) \mathrm{x}$ $80 \mu \mathrm{m}\left(2 t_{p}\right)$ and the developed microvalve dimensions are $4.8 \mathrm{~mm} \times 1.5 \mathrm{~mm} \times 1.5 \mathrm{~mm}$. Each dimension of the microvalve structure is designed to be limit to a maximum of $10 \mathrm{~mm}$ to maintain the miniaturized size of the device. Based on different microvalve structures that have been realised in the literature $[1,7,9,10,25]$, piezoelectric actuators are known for high force operation for a moderate input voltage as discussed in section 3.2. The longitudinal shape and the possibility to isolate the piezoelectric actuator from the fluid flow hence reduce environmental effects on the microvalve structure. By designing a piezoelectric diaphragm synchronized with the actuator, the microvalve can be developed into a micropump.

The microvalve is powered by a sinusoidal signal with an operating frequency $\left(f_{0}\right)$ of $50 \mathrm{~Hz}$ and a peak voltage $(V$ peak $)$ of $5 \mathrm{~V}$. Figure 6 depicts the side and top views of the microvalve structure and the placement of the bimorph actuator. Displacement of the actuator tip ( $\left.\mathbf{d}_{\text {tip }}\right)$ is theoretically calculated by:

$$
\mathbf{d}_{\text {tip }}=-\frac{3}{2} d_{31}\left(\frac{L}{t_{p}}\right)^{2} * \mathbf{V}, \quad \text { the input voltage } \mathbf{V}=V_{\text {peak }} * \operatorname{Sin}\left(2 \pi f_{0} t\right)
$$

Where $t$ is the time and $d_{31}$ is the piezoelectric constant relating the developed strain to the applied electric field. The simulated results for $\mathbf{d}_{\text {tip }}$ are obtained using ANSYS. PZT-4 material properties were used for both the theoretical and simulation analysis. 


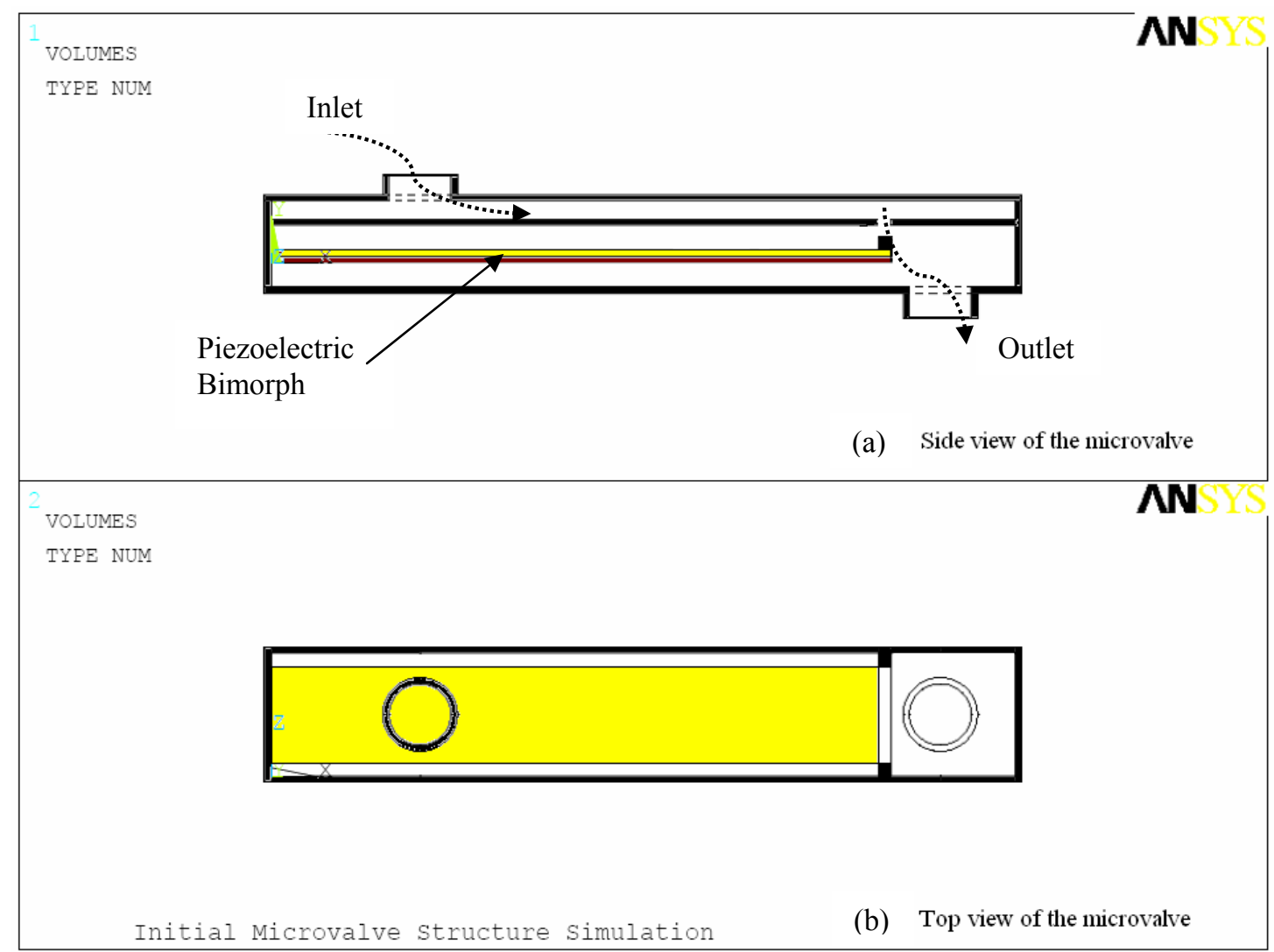

Figure 6. The microvalve structure Developed using ANSYS: (a) The side view of the structure which shows the path of the fluid flow when the valve is open, (b) The top view of the structure with the bimorph actuator

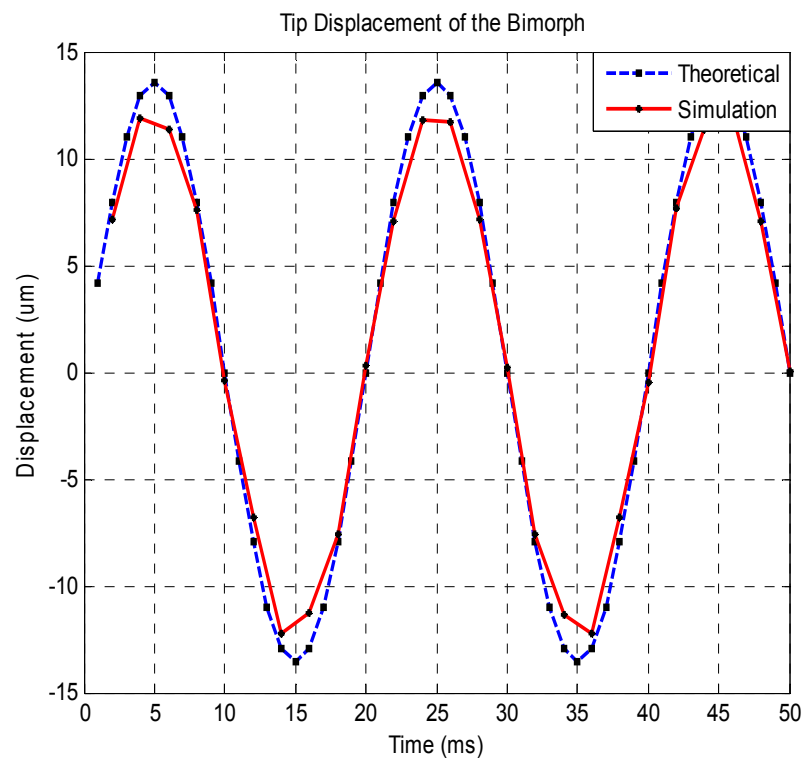

(a)

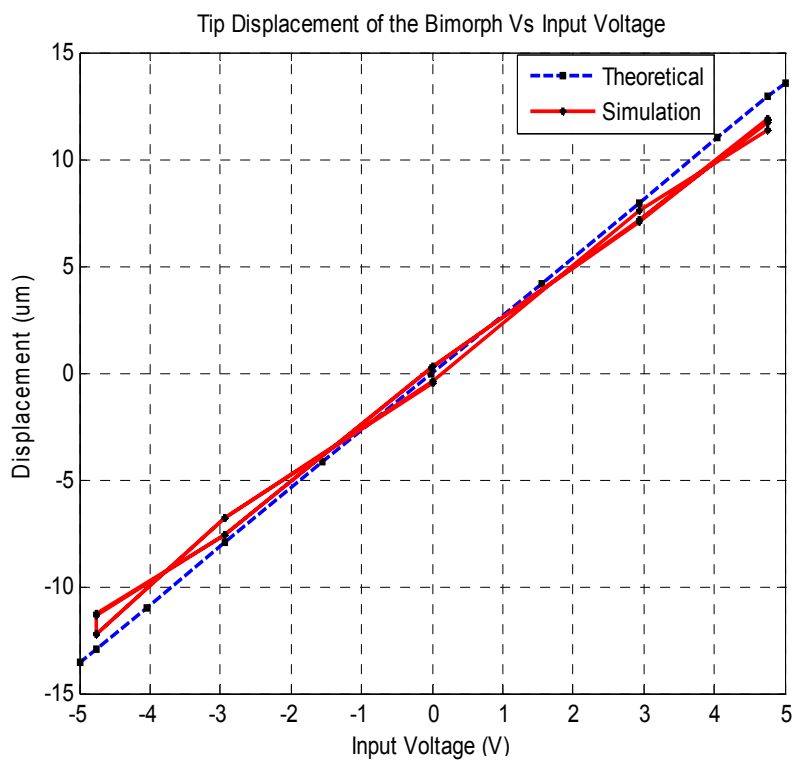

(b)

Figure 7. Comparison of Simulated and theoretical results for the developed microvalve structure: (a) Displacement Vs

Time plot for the simulated and theoretical tip displacement in bimorph actuator in the microvalve, (b)

Displacement Vs Input Voltage plot for the bimorph actuator. 
According to Figure 7, it can be seen that the simulated results agree with the theoretical results. Figure 7(b) shows the relation between the applied voltage and the achieved displacement of the microvalve. It can be seen that the voltage for the simulated plot varies between $\pm 4.75 \mathrm{~V}$ instead of $\pm 5 \mathrm{~V}$. This difference is mainly due to the two $1 \Omega$ resistors that are needed in the simulation to represent the wire and contact resistance in the device. Mainly due to the same reason, the simulated displacement tends to be slightly lower than the theoretical value as it can be noticed in both the Figures 7(a) and $7(b)$.

\section{CONCLUSION}

A biocompatible, battery-less and wirelessly controlled microvalve was discussed in this paper. It was identified that even though battery-less (passive) biomedical implants have been realised successfully, none of them known to have advance control with both sensing and actuating capability in the context of modulation of fluid flow. Therefore the need to develop a microvalve with battery-less operation with ensured biocompatibility has been regarded as a high requirement for most of the drug delivery and other similar applications such as fertility control and fast DNA sequencing.

A novel method based on using wireless communication and SAW devices for the interrogation of an implantable microvalve was highlighted. Power transfer from an interrogating RF signal was discussed as a successful alternative for the active operation of the microvalve. A review of different micropump structures was conducted and followed by an analysis of different actuation schemes. The importance of FEA in relation to constitutive equations was discussed and different acoustic modes were highlighted relevant to potential applications. Tradeoffs in antenna design were discussed and a preliminary analysis was carried out to calculate an appropriate interrogating frequency for the microvalve. Models for a SAW device and a microvalve were presented and a comparison of the performance of the microvalve was discussed using ANSYS tools.

\section{ACKNOWLEDGEMENT}

Authors would like to thank the Australian Research Council (ARC) and the School of Electrical and Electronic Engineering (University of Adelaide) for the funding and the support provided for this research.

\section{REFERENCES}

[1] P. J. Hesketh, J. S. Bintoro and R. Luharuka, "Microvalve for Fuel Cells and Miniature Gas Chromatographic System," Sensors Update, Vol. 13, pp 233-302, February 2004.

[2] W. G. Scanlon, J. B. Burns and N. E. Evans, "Radiowave Propagation form a Tissue-Implanted Source at 418 MHz and 916.5MHz," IEEE Transactions on Biomedical Engineering, Vol. 77, pp 527-534, April 2000.

[3] A. S. Sezen, S. Sivaramakrishnan, S. Hur, R. Rajamani, W. Robbins and B. J. Nelson, "Passive Wireless MEMS Microphones for Biomedical Applications," Journal of Biomedical Engineering, Vol. 127, pp 1030-1034, November 2005.

[4] I. Pitz, L.T. Hall, H.J. Hansen, V.K. Varadan, C.D. Bertram, S. Maddocks, S. Enderling, D. Saint, S.F. Al-Sarawi and D. Abbott, "Trade-offs for Wireless Transcutaneous RF Communication in Biotelemetric Applications," Proceedings of the SPIE, The International Society for Optical Engineering, Vol. 4937, pp 307-318, December 2002.

[5] M. Kohl, J. Gottert and J. Mohr, "Verification of the micromechanical characteristics of electrostatic linear actuators," Sensors and Actuators A, Vol. 53, no 1-3, pp 416-422, May 1996.

[6] M. Hamsch, "An Interrogation Unit for Passive Wireless SAW Sensors Based on Fourier Transform," IEEE Transactions on Ultrasonics, Ferroelectrics, and Frequency Control, Vol. 51, pp 1449-1455, November 2004.

[7] N. T. Nguyen, X. Huang and T. K. Chuan, "MEMS-Micropumps: A Review," Transactions of the ASME Journal of Fluids Engineering, Vol. 124, pp 384-392, June 2002.

[8] R. Duggirala, S. Son and A. Lal, "A Pyroelectric-Piezoelectric Valve for Integrated Microfluidics," IEEE International Solid-State Sensors and Actuators Conference, pp 1554-1557, June 2003.

[9] A. F. Ayhan, "Design of a Piezoelectrically Actuated Microvalve for Flow Control in Fuel Cells," Thesis submission for Masters of science in Mechanical Engineering-University of Pittsburgh, October 2002 
[10] K. W. Oh and C. H. Ahn, "A review of microvalves," Journal of Micromechanics and Microengineering, Vol. 16, pp 13-39, May 2006.

[11] V. K. Varadan and V. V Varadan, "Microsensors, micromechanical systems (MEMS), and electronics for smart structures and systems," Smart Materials and Structures, Vol. 9, pp 953-972, December 2000.

[12] V. V. Varadan, V. K. Varadan and X. Q. Bao, "IDT sensors for detection of ice on rotorcraft," Proceedings of the SPIE, The International Society for Optical Engineering, Vol. 3328, pp 49-58, March 1998.

[13] J. W. Gardner, V. K. Varadan and O. O. Awadelkarim, "Microsensors, Introduction to SAW Devics," Microsensors, MEMS, and Smart Devices, 2001.

[14] S. J. Ippolito, K. Kalantar-zadeh, W. Wlodarski and D. A. Powell, "Finite-element Analysis for Simulation of Layered SAW Devices with XY LiNbO3 Substrate," Proceedings of the SPIE, The International Society for Optical Engineering, Vol. 4935, pp 120-131, December 2002.

[15] F. Kubat, W. Ruile, T. Hesjedal, J.Stotz, U. Rosler and L. M. Reindl, "Calculation and experimental verification of the acoustic stress at GHz frequencies in SAW resonators," IEEE Transactions on Ultrasonics, Ferroelectrics, and Frequency Control, Vol. 51, pp 1437-1448, November 2004.

[16] K. Nakamura and S. Ito, "KNbO3 Single Crystals and Thin Films for SAW and BAW Devices," www.usl.chibau.ac.jp/ ken/Symp2004/PDF/1D1.PDF, last accessed on $20^{\text {th }}$ September 2006.

[17] Piezoelectric material properties and information, Engineering Fundamentals, http://www.efunda.com/materials/materials home/materials.cfm, last accessed on $19^{\text {th }}$ September 2006

[18] Lithium Niobate Crystal, Jiaxing Acoustic-Electric Industrial Company, http://www.sawseek.com/prody3.html last accessed on $19^{\text {th }}$ September 2006.

[19] ANSYS Incorporation official website, http://www.ansys.com/, last accessed $20^{\text {th }}$ September 2006.

[20] S. Chamaly, R. Lardat, T. Pastureaud, P. Dufilie, W. Steichen, O. Holmgren, M. Kuitunen, J. V. Knuuttila and M. M. Salomaa, "SAW Device Analysis Using a Combination of EM/BEM Calculations and Scanning Interferometer Measurements," IEEE Ultrasonic Symposium, IEEE Ca-No. 03CH37476, Vol. 1, pp 294-298, October 2003.

[21] Chemical Definitions, http://www.chemicool.com/definition/epitaxial.html, last accessed $20^{\text {th }}$ September 2006.

[22] P. Soontornpipit, C. M. Furse and Y. C. Chung, "Design of Implantable Microstrip Antenna for Communication with Medical Implants," IEEE Transactions on Microwave Theory and Techniques, Vol. 52, pp 1944-1951, August 2004.

[23] W. G. Scanlon and N. E. Evans, "Numerical analysis of bodyworn UHF antenna systems," Electronics and Communication Engineering Journal, Vol. 13, pp 53-64, April 2001.

[24] ICNIRP Guidelines, "Guidelines for limited exposure to time-varying electric, magnetic, and electromagnetic fields (up to $300 \mathrm{GHz}$ )," International Commission on Non-Ionizing Protection, Health Phys, Vol. 75, pp 494522, April 1998.

[25] T. Morita, T. Niino, H. Asama and H. Tashiro, "Fundamental Study of a Stacked Lithium niobate Transducer," Japanese Journal of Applied Physics, Vol. 40, pp 3801-3806, May 2001.

[26] Technical Publication TP-223- Ceramic Bender Bimorphs, Morgan Electro Ceramics, http://www.morganelectroceramics.com/, last accessed $25^{\text {th }}$ September 2006.

[27] Technical Publication TP-245- Cantilever Mounted PZT 5A Bimorphs ${ }^{\circledR}$, Morgan Electro Ceramics, http://www.morganelectroceramics.com/, last accessed $25^{\text {th }}$ September 2006. 